\title{
Predicting fluorescence quantum yields for molecules in solution: A critical assessment of the harmonic approximation and the choice of the lineshape function
}

\author{
Alexander Humeniuk, ${ }^{1}$ Margarita Bužančić, ${ }^{2}$ Joscha Hoche, ${ }^{1}$ Javier Cerezo, ${ }^{3}$ Roland Mitrić, ${ }^{1,}$ a) Fabrizio \\ Santoro, 4, b) and Vlasta Bonačić-Koutecký2, 5, c) \\ ${ }^{1)}$ Institut für Physikalische und Theoretische Chemie, Universität Würzburg, Am Hubland, 97074 Würzburg, \\ Germany. \\ ${ }^{2)}$ Center of Excellence for Science and Technology-Integration of Mediterranean region (STIM), Faculty of Science, \\ University of Split, Poljička cesta 35, 2100 Split, Croatia. \\ 3) Departamento de Química, Facultad de Ciencias, Universidad Autónoma de Madrid, 28049 Madrid, \\ Spain. \\ ${ }^{4)}$ Istituto di Chimica dei Composti Organometallici (ICCOM-CNR), Area della Ricerca del CNR, Via Moruzzi 1, \\ I-56124 Pisa, Italy. \\ ${ }^{5)}$ Department of Chemistry, Humboldt Universität zu Berlin, Brook-Taylor-Strasse 2, 12489 Berlin, \\ Germany.
}

For the rational design of new fluorophores, reliable predictions of fluorescence quantum yields from first principles would be of great help. However, efficient computational approaches for predicting transition rates usually assume that the vibrational structure is harmonic. While the harmonic approximation has been used successfully to predict vibrationally resolved spectra and radiative rates, its reliability for nonradiative rates is much more questionable. Since non-adiabatic transitions convert large amounts of electronic energy into vibrational energy, the highly excited final vibrational states deviate greatly from harmonic oscillator eigenfunctions. We employ a time-dependent formalism to compute radiative and non-radiative rates for transitions and study the dependence on model parameters. For several coumarin dyes we compare different adiabatic and vertical harmonic models (AS, ASF, AH, VG, VGF, VH), in order to dissect the importance of displacements, frequency changes and Duschinsky rotations. In addition we analyze the effect of different broadening functions (Gaussian, Lorentzian or Voigt). Moreover, to assess the qualitative influence of anharmonicity on the internal conversion rate, we develop a simplified anharmonic model. We adress the reliability of these models considering the potential errors introduced by the harmonic approximation and the phenomenological width of the broadening function.

\section{INTRODUCTION}

Luminescence has many technological applications such as organic light-emitting diodes, luminescent solar concentrators $^{1}$ and in vivo fluorescence imaging. ${ }^{2}$ The high cost for synthesizing and characterizing new molecules has revived the interest in reliable prediction of fluorescence and phosphorescence quantum yields (QY) from first principles. To identify candidate molecules for synthesis, virtual screenings could be performed for identifying the most promising novel compounds with high quantum yields and other desirable properties such as low emission energy in the infrared part of the spectrum. Recently several programs ${ }^{3,4}$ were developed for predicting rates for radiative and non-radiative (internal conversion, $\mathrm{IC}^{5,6}$, and intersystem crossing, $\mathrm{ISC}^{7,8}$ ) decays using Fermi's Golden rule. They are based on the idea that the decay processes are slower than thermalization and that the couplings are small. In addition, all of these approaches make use of the harmonic approximation for the ground- and excited-state potential energy surfaces

\footnotetext{
a) Electronic mail: roland.mitric@uni-wuerzburg.de

b) Electronic mail: fabrizio.santoro@pi.iccom.cnr.it

c) Electronic mail: vbk@chemie.hu-berlin.de
}

(PES), since this is at the moment the only way how to deal with systems of interest having dozens or hundreds of normal modes. Some of the methods can account for mixing of modes (Duschinsky rotation), the Herzberg-Teller correction and temperature dependence. The scope of this article is to investigate the range of validity of these state of the art methodologies. Despite some contributions in the literature ${ }^{9}$, this question is not yet well assessed.

Differences between simulated IC rates based on Fermi's Golden rule and experimental non-radiative rates can have many origins: additional decay channels (ISC or conical intersections, dark trap states), additional effects that were not considered (Duschinsky or HerzbergTeller effect), or simply a questionable description of the electronic structure. These issues can be treated by more advanced harmonic models that take all relevant vibronic effects into account or by including additional rate constants for pronounced non-harmonic processes, such as thermally activated decay through a conical intersection. ${ }^{10,11}$ But what about the harmonic approximation itself, on which the whole theory rests? The harmonic approximation is the central issue for calculations of radiative and internal conversion rates, in particular if one is interested in large systems. All kinds of transitions (radiative, IC, ISC, charge transfer) can be 
formulated in terms of Fermi's Golden rule making use of the harmonic approximation. ${ }^{3,12}$ However, the approximation is not equally applicable to all transitions. Radiative rates are usually determined by transitions between vibrational ground states or states with few vibrational quanta. Intersystem crossing is most effective if the singlet and triplet states are almost degenerate, so that the ISC rate is also dominated by transitions between vibrational ground states. On the other hand, internal conversion involves transitions to highly excited vibrational final states (see Fig. 1). Electronic energy of a few electron volts is converted into vibrational energy, which is enough to overcome the barriers that separate different isomers on the ground-state PES. These considerations should be taken as warnings regarding the use of the harmonic approximation. However, it is the only practical approach because of the convenient form of multidimensional Gaussian integrals and it is very difficult to go beyond it.

To test how well the harmonic approximation is suited for predicting fluorescence quantum yields, we study a series of coumarin dyes for which experimental non-radiative rates and quantum yields are available. We use a quite general time-dependent formalism ${ }^{13}$ to compute rates as Fourier transforms of correlation functions. Density functional theory in combination with a polarizable continuum model (PCM) for solvation provides the vibrational structure of the initial and final electronic states. Since only a few standard electronic structure calculations are required and the time-dependent formalism is very efficient, this approach for ab initio prediction of fluorescence quantum yields seems attractive.

But the question remains whether the harmonic approximation is valid and how we can test its validity without knowing the exact solution. For $1 \mathrm{D}$ and $2 \mathrm{D}$ systems the exact solution can be obtained, but such low-dimensional systems are not relevant models for typical chromophores which contain dozens to a hundred vibrational modes, whose frequencies span the range from $<50 \mathrm{~cm}^{-1}$ to $>3500 \mathrm{~cm}^{-1}$.

Since in principle molecular PES are not harmonic, one has to choose a point at which the quadratic approximation is made. This choice defines different harmonic models. ${ }^{14}$ The models can be separated into two classes, depending on around which point the potential energy surface of the electronic ground state is expanded to second order. In adiabatic models $(\mathrm{AS} / \mathrm{ASF} / \mathrm{AH})$ the potential is expanded around the geometry of the ground-state minimum, while in vertical models $(\mathrm{VG} / \mathrm{VGF} / \mathrm{VH})$ the surface is expanded around the excited-state minimum. The models can be further distinguished depending on whether the initial and final states have different equilibrium geometries, but the same normal modes (adiabatic shift AS, vertical gradient VG), differ in equilibrium geometry and frequencies (adiabatic shift frequencies ASF, vertical gradient frequencies VGF) or differ in equilibrium geometry and the Hessian matrix (adiabatic Hessian $\mathrm{AH}$, vertical Hessian $\mathrm{VH}$ ).
In this contribution we compare the performance of these models. There is a clear hierarchy with those higher up being in principle more accurate: $\mathrm{AS}<\mathrm{ASF}<\mathrm{AH}$ and $\mathrm{VG}<\mathrm{VGF}<\mathrm{VH}$. Although $\mathrm{AH}$ and $\mathrm{VH}$ are the best models, retaining inferior ones has the advantage that we can decompose the non-radiative rate into contributions from different effects. Moreover, since $\mathrm{AH}$ and $\mathrm{VH}$ models should deliver identical results if harmonic approximation is exact we will inquire to what extent an analysis of their difference is a trustworthy reporter of the importance of anharmonic effects.

As a second step of our analysis, in this contribution we explicitly tackle the study of possible anharmonic effects on the internal conversion rate by considering simple models. More specifically, for this purpose we formulate a model that interpolates smoothly between the harmonic approximation and a fictitious anharmonic system. ${ }^{15}$ Realistic harmonic frequencies, displacements and electronic non-adiabatic coupling vectors are taken from a TD-DFT + PCM calculation. These are the same quantities needed for the adiabatic shift (AS) model, where ground- and excited-state PES differ only by a vertical shift in energy and a horizontal shift of the equilibrium geometries. To incorporate anharmonicity each normal mode is replaced by a Morse potential that is characterized by an anharmonicity $\chi$. For $\chi=0$ the Morse potential turns into a harmonic potential with the same frequency and equilibrium position as the corresponding harmonic potential. $\chi$ is a global parameter, it is the same for all modes. For $\chi>0$ the modes acquire some anharmonicity. Since the dissociation energy $D$ of a Morse potential with frequency $\omega$ is related to the anharmonicity via $D=\frac{\hbar \omega}{4 \chi}$, modes with low frequencies are more affected.

It is important to note that the anharmonic model is not a realistic representation of any molecule for a number of reasons: Low-lying modes such as rotations do not have the form of a Morse potential; the modes are assumed to be independent of each other and all other approximations made in the AS model, except for the harmonicity of the potential, still apply. With these caveats in mind we investigate how the rate of internal conversion in 3chloro-7-methoxy-4-methylcoumarin, CIMMC, changes with the anharmonicity $\chi$.

The paper is structured in the following way: In section II we sketch the time-dependent formalism for obtaining transition rates in the harmonic approximation and derive the anharmonic AS model. Section III gives the computational details. In section IV we present vibrationally resolved emission spectra, non-radiative rates and quantum yields for a selection of coumarin dyes, focusing on the influence of anharmonicity, choice of the harmonic model, and broadening function. 

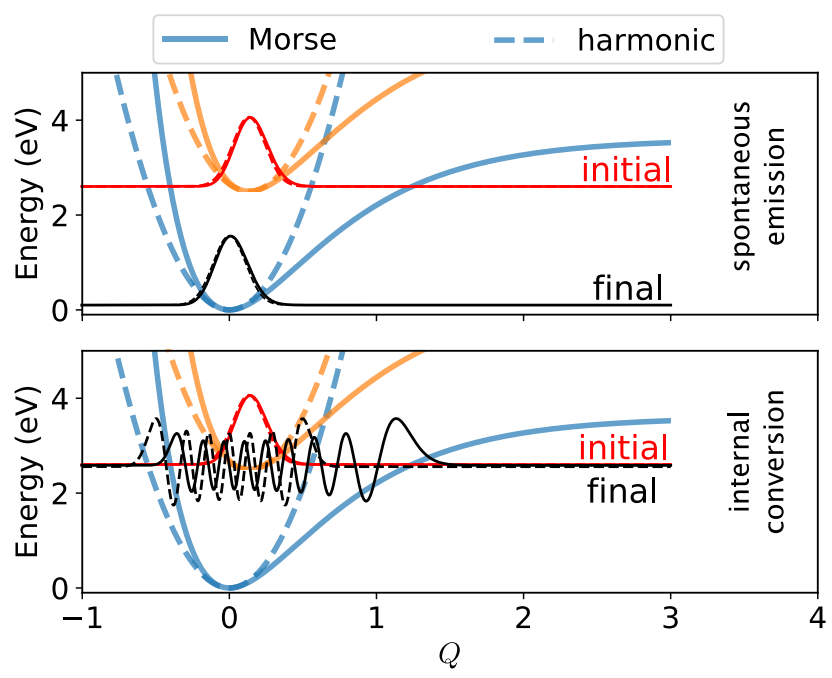

FIG. 1. Morse potential for a $\mathrm{C}=\mathrm{C}$ stretching mode. The initial and final states for radiative (top) and non-radiative (bottom) transitions are also shown both for the Morse potential (anharmonicity $\chi=0.014$, solid lines) and the harmonic approximation $(\chi=0.0$, dashed lines). The radiative rate is dominated by the transition between two vibrational ground states, for which the harmonic approximation is valid. With perfect energy conservation the non-radiative rate is determined by the transition between a vibrational ground state on the excited state PES and a highly excited vibrational state on the ground state PES. The wavefunction of the final vibrational state differs considerably from an harmonic oscillator wavefunction. However, the overlap between initial and final state is very low due to the narrow oscillations in both the Morse and the harmonic oscillator wavefunction.

\section{THEORY}

\section{A. Harmonic Models - Transition Rates in the Time-Dependent Formalism}

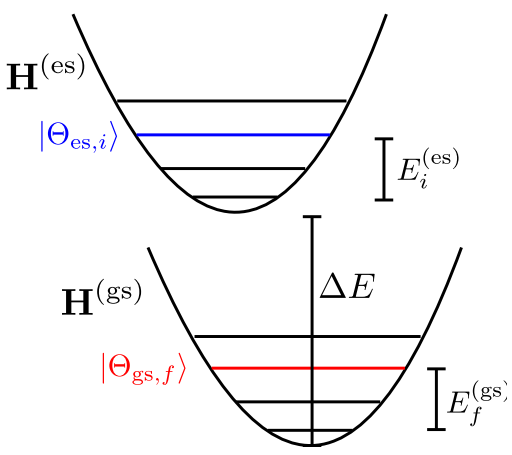

FIG. 2. Harmonic potential energy surfaces, adiabatic excitation energy $\Delta E$ and vibrational energies of an initial and a final state.

Non-radiative transitions are treated with first order perturbation theory. We invoke the Born-Oppenheimer approximation so that the initial (excited) and the final (ground) state can be expressed as products of an electronic $(\Psi)$ and a vibrational $(\Theta)$ wavefunction. The nonadiabatic transition is mediated by the nuclear kinetic energy operator $\hat{V}=\hat{T}_{\text {nuc }}$ :

$$
\left|\Psi_{\mathrm{es}}, \Theta_{\mathrm{es}, i}\right\rangle \stackrel{\hat{V}}{\longrightarrow}\left|\Psi_{\mathrm{gs}}, \Theta_{\mathrm{gs}, f}\right\rangle
$$

We further assume that the probability to be initially in state $i$ is given by the Boltzmann distribution with partition function $Z=\sum_{i} e^{-\beta E_{i}^{(\mathrm{es})}}$ with $\beta=1 /\left(k_{\mathrm{B}} T\right)$. The internal conversion rate is then given by Fermi's Golden rule:

$$
\begin{aligned}
k_{\mathrm{ic}}(\Delta E)=\frac{2 \pi}{\hbar} & \sum_{i, f} \frac{e^{-\beta E_{i}^{(\mathrm{es})}}}{Z}\left|\left\langle\Psi_{\mathrm{es}}, \Theta_{\mathrm{es}, i}|\hat{V}| \Psi_{\mathrm{gs}}, \Theta_{\mathrm{gs}, f}\right\rangle\right|^{2} \\
& \times \delta\left(\Delta E+E_{i}^{(\mathrm{es})}-E_{f}^{(\mathrm{gs})}\right)
\end{aligned}
$$

For the definition of the vibrational energies $E_{i}^{(\mathrm{es})}$ and $E_{f}^{(\mathrm{gs})}$ and the adiabatic excitation energy $\Delta E$ see Fig. 2. Enumerating and summing over all relevant vibrational states in even a medium-sized molecule is very difficult. In the time-dependent formalism this problem is solved by replacing the summation by a propagation in time. The lineshape function $\delta(\ldots)$, which enforces energy conservation, is replaced by its Fourier decomposition:

$\delta\left(\Delta E+E_{i}^{(\mathrm{es})}-E_{f}^{(\mathrm{gs})}\right)=\frac{1}{2 \pi} \int_{-\infty}^{\infty} e^{\imath\left(\Delta E+E_{i}^{(\mathrm{es})}-E_{f}^{(\mathrm{gs})}\right) t} \tilde{f}(t) d t$

Here $\tilde{f}(t)$ is the Fourier transform of the lineshape function and accounts for homogeneous and inhomogeneous broadening. In this way the Fourier transform of the rate is expressed formally as a time correlation function:

$$
\tilde{k}_{\mathrm{ic}}(t)=\frac{2 \pi}{\hbar} \frac{1}{Z} \operatorname{Tr}\left(e^{(\imath t-\beta) \hat{H}^{(\mathrm{es})}} \hat{V}_{e, g} e^{-\imath t \hat{H}^{(\mathrm{gs})}} \hat{V}_{e, g}^{\dagger}\right)
$$

$\hat{H}^{(\mathrm{es})}$ and $\hat{H}^{(\mathrm{gs})}$ are the vibrational Hamiltonians of the excited and ground state, respectively and $\hat{V}_{e, g}=$ $\left\langle\Psi_{\mathrm{es}}|\hat{V}| \Psi_{\mathrm{gs}}\right\rangle$ is the matrix element of the coupling operator between the electronic parts of the wavefunctions. The rate is obtained by the inverse Fourier transform:

$$
k_{\mathrm{ic}}(\Delta E)=\frac{1}{2 \pi} \int_{-\infty}^{\infty} d t e^{\imath \Delta E t} \tilde{f}(t) \tilde{k}_{\mathrm{ic}}(t)
$$

The same formalism can also be applied to the radiative rate. Evaluating the correlation function in Eqn. 4 is still a formidable task, unless the vibrational Hamiltonians $\hat{H}^{(\mathrm{es})}$ and $\hat{H}^{(\mathrm{gs})}$ are approximated by harmonic oscillators. For the general case of two harmonic potential energy surfaces the working equations for computing radiative and non-radiative rates have been derived in Refs. ${ }^{3,13,16,17}$. The reader is referred to these publications for more details. The formalism can deal with the changes of frequencies and mixing of normal modes 
(Duschinsky effect) and only requires standard electronic structure calculations. Geometry optimizations and frequency calculations can be routinely applied to excited states of medium-sized molecules. Two of us, J. Cerezo and F. Santoro, have implemented the time-dependent formalism into a development version of the code $F C$ classes $3^{4}$.

To study the effect of anharmonicity we modify the simplest of the harmonic models, the AS model, by replacing the quadratic potentials with Morse potentials. We now describe the anharmonic AS model and derive the corresponding expressions for the non-radiative rate.

\section{B. The Anharmonic Adiabatic Shift Model - Internal Conversion Rates}

An adiabatic shift (AS) model for internal conversion is fully characterized by the adiabatic excitation energy $\Delta E$, the vibrational frequencies $\omega_{i}$, the displacements $\Delta Q_{i}$ and the electronic non-adiabatic couplings between the ground and excited electronic states, $C_{i}=$ $\left\langle\Psi_{\mathrm{es}} \mid \frac{\partial \Psi_{\mathrm{gs}}}{\partial Q_{i}}\right\rangle$, for each of the independent vibrational modes $Q_{i}(i=1, \ldots, N)$. Using these quantities we construct potential energy surfaces for the electronic ground and excited state, where the harmonic oscillators for both the ground and excited states of a mode $i$ are replaced by Morse potentials ${ }^{18,19}$,

$$
\begin{aligned}
& V_{\mathrm{gs}}^{(i)}\left(Q_{i}\right)=D_{i}\left(1-e^{-\alpha_{i} Q_{i}}\right)^{2} \\
& V_{\mathrm{es}}^{(i)}\left(Q_{i}\right)=D_{i}\left(1-e^{-\alpha_{i}\left(Q_{i}-\Delta Q_{i}\right)}\right)^{2}+\Delta E .
\end{aligned}
$$

The ground- and excited-state minima lie at $Q_{i}=0$ and $Q_{i}=\Delta Q_{i}$, respectively. Around them the potential energy surfaces can be approximated by a harmonic oscillator potential with frequency $\omega_{i}$ :

$$
\begin{aligned}
V_{\mathrm{gs}}^{(i)}\left(Q_{i}\right) & \approx \frac{1}{2} \omega_{i}^{2} Q_{i}^{2} \\
V_{\mathrm{es}}^{(i)}\left(Q_{i}\right) & \approx \frac{1}{2} \omega_{i}^{2}\left(Q_{i}-\Delta Q_{i}\right)^{2}+\Delta E
\end{aligned}
$$

The parameters $D_{i}$ and $\alpha_{i}$ in the definition of the Morse potential can be expressed in terms of the frequency $\omega_{i}$ and an anharmonicity parameter $\chi$ :

$$
\begin{aligned}
D_{i} & =\frac{\omega_{i}}{4 \chi} \\
\alpha_{i} & =\sqrt{2 \omega_{i} \chi}
\end{aligned}
$$

For $\chi \rightarrow 0$, the Morse potentials turn into the harmonic oscillator potentials of Eqs. 8 and 9. The timeindependent Schrödinger equation,

$$
-\frac{\hbar^{2}}{2} \frac{\partial^{2} \phi^{(i)}}{\partial Q^{2}}+V^{(i)}(Q) \phi_{n}^{(i)}(Q)=\epsilon \phi_{n}^{(i)}(Q)
$$

is exactly solvable for the Morse potential leading to bound states with the eigenenergies

$\epsilon_{n}^{(i)}=\hbar \omega_{i}\left(\left(n+\frac{1}{2}\right)-\chi\left(n+\frac{1}{2}\right)^{2}\right) \quad n=0,1, \ldots, n_{\max }$.

The index of the highest vibrational state $n_{\max }$ which is still bound can be determined by the requirement that the energy increases monotonically with the index $n$, therefore

$$
\left.\frac{\partial \epsilon}{\partial n}\right|_{n=n_{\max }}=0 \Rightarrow n_{\max }=\left\lfloor\frac{1}{2}\left(\frac{1}{\chi}-1\right)\right\rfloor .
$$

The main difference between the harmonic and the Morse potential is that in the latter most states are unbound, so that the density of final states is increased if the adiabatic excitation energy exceeds the dissociation energy.

We solve the Schrödinger equation for the ground- and excited-state Morse potential numerically for each mode on an equidistant grid by diagonalizing a finite difference Hamiltonian. The overlap integrals between eigenfunctions on the excited state $\left(n_{i}\right)$ and the ground state $\left(m_{i}\right),\left\langle n_{i}^{\prime} \mid m_{i}\right\rangle=\int d Q_{i} \phi_{n}^{(i)}\left(Q_{i}-\Delta Q_{i}\right)^{*} \phi_{m}^{(i)}\left(Q_{i}\right)$, as well as the nuclear non-adiabatic coupling vectors, $\left\langle n_{i}^{\prime} \mid \nabla m_{i}\right\rangle=$ $\int d Q_{i} \phi_{n}^{(i)}\left(Q_{i}-\Delta Q_{i}\right)^{*} \frac{\partial \phi_{m}^{(i)}\left(Q_{i}\right)}{\partial Q_{i}}$ are evaluated numerically and tabulated. The fact that the eigenenergies are known exactly and that the Franck-Condon factors should sum to $\sum_{m_{i}=0}\left|\left\langle n_{i}^{\prime} \mid m_{i}\right\rangle\right|^{2}=1$ serves as a check.

A multidimensional potential energy surface (for the ground or excited state) is constructed as a sum of the $N$ Morse potentials:

$$
V\left(Q_{1}, Q_{2}, \ldots, Q_{N}\right)=\sum_{i=1}^{N} V^{(i)}\left(Q_{i}\right)
$$

A vibrational wavefunction is specified by the number of phonons $m_{i}$ in each mode

$$
|\vec{m}\rangle=\left|m_{1}, m_{2}, \ldots, m_{N}\right\rangle,
$$

with eigenenergies

$$
E_{\vec{m}}=\sum_{i=1}^{N} \epsilon_{m_{i}}^{(i)}
$$

Vibrational relaxation in the excited state is taken to be very fast as compared to electronic relaxation. Therefore the probability of being in the initial vibrational state $\vec{n}$ is given by the Boltzmann distribution $P_{\vec{n}}=\frac{e^{-\beta E_{\vec{n}}}}{Z}$.

The total rate of internal conversion is the sum over all transitions between initial states $\Psi_{\mathrm{es}} \Theta_{\mathrm{es}, \vec{n}}$ and final states $\Psi_{\mathrm{gs}} \Theta_{\mathrm{gs}, \vec{m}}$ weighted by the probability $P_{\vec{n}}$ :

$$
\begin{aligned}
k_{\mathrm{ic}}(\Delta E)=2 \pi & \sum_{\vec{n}, \vec{m}} P_{\vec{n}}\left|\sum_{i}\left\langle\Psi_{\mathrm{gs}} \mid \frac{\partial \Psi_{\mathrm{es}}}{\partial Q_{i}}\right\rangle\left\langle\Theta_{\mathrm{gs}, \vec{m}} \mid \frac{\partial \Theta_{\mathrm{es}, \vec{n}}}{\partial Q_{i}}\right\rangle\right|^{2} \\
& \times \delta\left(\Delta E+E_{\vec{n}}-E_{\vec{m}}\right)
\end{aligned}
$$


$\delta(\ldots)$ enforces (approximate) energy conservation between initial and final states, $\Delta E+E_{\vec{n}} \approx E_{\vec{m}}$. In the time-dependent formalism the non-radiative rate is expressed as

$$
k_{\mathrm{ic}}(\Delta E)=\frac{1}{2 \pi} \int_{-\infty}^{+\infty} e^{\imath \Delta E t} \tilde{f}(t) \tilde{k}_{\mathrm{ic}}(t) d t
$$

where $\tilde{f}(t)$ is the Fourier transform of the lineshape function (Gaussian $\tilde{f}_{\mathrm{G}}(t)=1 /(2 \pi) \exp \left(-1 / 2 \sigma^{2} t^{2}\right)$, Lorentzian $\tilde{f}_{\mathrm{L}}(t)=1 /(2 \pi)[\exp (\gamma t) \theta(-t)+\exp (-\gamma t) \theta(t)]$ with $\theta(t)$ the Heaviside step function, or a Voigt profile $\left.\tilde{f}_{\mathrm{V}}(t)=\tilde{f}_{\mathrm{G}}(t) \tilde{f}_{\mathrm{L}}(t)\right)$ and $\tilde{k}_{\mathrm{ic}}(t)$ is a correlation function:

$$
\tilde{k}_{\mathrm{ic}}(t)=u_{\mathrm{ic}} Z^{-1} \sum_{\vec{n}} e^{-\beta E_{\vec{n}}} \sum_{\vec{m}} F_{\vec{n}, \vec{m}} N_{\vec{n}, \vec{m}}^{2} e^{-\imath\left(E_{\vec{m}}-E_{\vec{n}}\right) t}
$$

The factor $u_{\text {ic }}=2.598 \times 10^{17} \mathrm{~s}^{-1}$ converts the rate to $\mathrm{s}^{-1}$ if all other quantities are given in atomic units. The sum extends over all vibrational initial and final states with occupation numbers $\vec{n}$ and $\vec{m}$, respectively. $F_{\vec{n}, \vec{m}}$ is the product of Franck-Condon factors $\mathrm{FCF}_{n_{i}, m_{i}, i}=$ $\left|\left\langle n_{i}^{\prime} \mid m_{i}\right\rangle\right|^{2}$

$$
F_{\vec{n}, \vec{m}}=|\langle\vec{n} \mid \vec{m}\rangle|^{2}=\prod_{i=1}^{N} \mathrm{FCF}_{n_{i}, m_{i}, i}
$$

and $N_{\vec{n}, \vec{m}}$ is the scalar product of the electronic with the nuclear non-adiabatic coupling vector (divided by the overlap)

$$
\begin{aligned}
N_{\vec{n}, \vec{m}} & =\sum_{i=1}^{N}\left\langle\Psi_{\mathrm{es}} \mid \frac{\partial \Psi_{\mathrm{gs}}}{\partial Q_{i}}\right\rangle \cdot \frac{\left\langle n_{i}^{\prime}\left|\frac{\partial}{\partial Q_{i}}\right| m_{i}\right\rangle}{\left\langle n_{i}^{\prime} \mid m_{i}\right\rangle} \\
& =\sum_{i} C_{i} \cdot K_{n_{i}, m_{i}, i}
\end{aligned}
$$

To bring Eqn. 20 into a manageable form we define

$$
G_{n, m, i}(\beta, t)=e^{-\beta \epsilon_{n}^{(i)}} F_{n, m, i} e^{-\imath\left(\epsilon_{m}^{(i)}-\epsilon_{n}^{(i)}\right) t}
$$

and sums over products $G_{n, m, i}$ with the non-adiabatic couplings $K_{n, m, i}$

$$
\begin{aligned}
& g_{i}^{(0)}(\beta, t)=\sum_{m} \sum_{n} G_{m, n, i}(\beta, t) \\
& g_{i}^{(1)}(\beta, t)=\sum_{m} \sum_{n} G_{m, n, i}(\beta, t) K_{n, m, i} \\
& g_{i}^{(2)}(\beta, t)=\sum_{m} \sum_{n} G_{m, n, i}(\beta, t) K_{n, m, i}^{2} .
\end{aligned}
$$

For a harmonic oscillator these sums can be obtained analytically, in the case of the Morse potentials the sum runs over all eigenstates, whose number is limited by the size of the grid that was used to calculate them.

It can be shown that with these abbreviations the correlation function becomes

$$
\tilde{k}_{\mathrm{ic}}(t)=u_{\mathrm{ic}} Z^{-1}\left\{\sum_{i=1}^{N} \sum_{j=1}^{N} g_{i}^{(1)}(\beta, t) g_{j}^{(1)}(\beta, t) \prod_{\substack{k \\ k \neq i \\ k \neq j}} g_{k}^{(0)}(\beta, t)+\sum_{i=1}^{N} g_{i}^{(2)}(\beta, t) \prod_{\substack{k \\ k \neq i}} g_{k}^{(0)}(\beta, t)\right\}
$$

\section{COMPUTATIONAL DETAILS}

For the simulation of vibrationally resolved fluorescence spectra of the studied systems, the molecular structure has been first optimized in the framework of density functional theory (DFT), using the PBE0 hybrid functional (25\% HF exchange $)^{20,21}$ and the B3LYP hybrid functional $^{22}$ in combination with the def2-SVP ${ }^{23}$ basis set as implemented in the Gaussian $16^{24}$ software package. The PBE0 functional was employed for the main part of the analysis because it has been found to give reasonably accurate spectral lineshapes in previous studies of coumarin dyes. ${ }^{25,26}$ The excited state properties have been calculated using time-dependent density functional theory (TD-DFT) with the same functional and basis set. The solvent effects are treated in an implicit way via the polarizable continuum model (PCM). ${ }^{27}$ For the calculation of excited state properties, the reaction field of the solvent is adjusted to the electronic density of the first excited state (equilibrium solvation). The vibrationally resolved emission spectra together with radiative and non-radiative rates from the lowest electronic excited state were simulated within the harmonic and FranckCondon approximations employing the FCclasses $3^{4}$ program.

The internal conversion rates based on the anharmonic AS model are calculated for a range of anharmonicity parameters, $\chi=\{0.0,0.01,0.02,0.03,0.04,0.05,0.06,0.07\}$. The correlation function $\tilde{k}_{\text {ic }}(t)$ of Eqn. 27 is evaluated on an equidistant time grid covering the interval $[-T, T]$ for $T=1000$ fs with $N_{t}=2^{14}+1$ samples. $\tilde{k}_{\text {ic }}(t)$ is Fourier transformed followed by interpolation on the discrete energy grid to obtain the rate $k_{\text {ic }}(\Delta E)$ at the emission energy $\Delta E$. 


\section{RESULTS}

To test whether we can predict fluorescence quantum yields using harmonic models, we selected a sample of coumarin derivatives, for which experimental quantum yields in solution are available in the literature ${ }^{28-33}$. Coumarin derivatives are versatile laser dyes ${ }^{34}$ and photosensitizers in solar cells and have been studied before by some of us. ${ }^{35}$ Interestingly coumarin itself does not fluoresce presumably because of a conical intersection that opens an ultrafast decay channel to the ground state through ring cleavage. ${ }^{36}$ In other coumarin derivatives fluorescence is diminished by the presence of a dark $n \pi^{*}$ state below the photoexcited $\pi \pi^{*}$ state, whose relative position depends on the polarity of the solvent. ${ }^{36}$ Since we want to study the validity of the harmonic approximation separately from other problems that might occur, we selected the coumarins 102, 343, 522 and ClMMC, which are well-behaved from the theoretical point of view: They do not have low-lying accessible conical intersections. Their $\mathrm{S}_{1}$ state is bright and of $\pi \pi^{*}$ character and is separated by a large energy gap from higher states, so that no mixing of states and breakdown of the BornOppenheimer approximation has to be considered. In the coumarins 102 and $\mathbf{3 4 3}$ the amine group is rigidized by six-membered rings, ${ }^{34}$ which impedes pyramidalization and rotation.

\section{A. Harmonic Models and Broadening Functions}

First we compare different harmonic models. In Fig. 3 (and Figs. 1 and 2 in the supplementary material) we present emission spectra, radiative and internal conversion rates for all coumarins. Comparing vertical $\left(\mathrm{V}^{*}\right)$ and adiabatic $\left(\mathrm{A}^{*}\right)$ models gives us the opportunity to assess the degree of anharmonicity, because corresponding adiabatic and vertical models should give the same results for a strictly harmonic potential. In this case the point around which the potential is expanded to second order becomes irrelevant. The agreement between vertical and adiabatic models, however, is only a necessary but not sufficient condition for the validity of the harmonic approximation, since the models become identical if the shift between the initial and final state minima is very small.

The accuracy of the harmonic approximation for the radiative decay rate $k_{\mathrm{r}}$ is remarkable (see Table I). For all cases in which experimental data is available our computations agree within a factor of 2, except $\mathrm{AH}$ value for ClMMC in cyhex which is $37 \%$ of the experimental value. The $\mathrm{VH}$ value for coumarin 102 in $\mathrm{MeCN}$ is only $7 \%$ smaller than what is measured in experiment. The emission spectra are narrow and structured for AS and VG models and are broadened and shifted to lower energies for $\mathrm{VH}$ and $\mathrm{AH}$ models. Therefore the radiative rate, $k_{\mathrm{r}}$, is also larger for $\mathrm{AS}$ and VG models since the rate scales as $\Delta E^{3}$. Differences in the predictions of $\mathrm{AH}$ and $\mathrm{VH}$ models are almost in all cases very minor, except for CIMMC in cyhex where a factor of 1.7 is observed. This is not unexpected since $k_{\mathrm{r}}$ mainly depends on the overlap between the thermally-populated vibrational states of the excited states (few quanta) and the low-energy vibrational states of the ground-electronic state (due to the $\Delta E^{3}$ dependence). These states should be well described in the harmonic approximation for rigid molecule as the coumarins here considered.

Moving to the predictions for internal conversion rate, for all coumarins they increase if frequency changes and Duschinsky mixings are taken into account. The rates of all harmonic models follow the exponential energy gap law, $k_{\text {ic }}(\Delta E) \propto e^{-\alpha \Delta E}$, yet the exponent depends on the model. Interestingly, also for $k_{\text {ic }} \mathrm{AH}$ and $\mathrm{VH}$ predictions are always similar, even in those cases in which $k_{\text {ic }}$ is very small, order of magnitudes less than what is necessary for a reliable prediction of the fluorescence quantum yield (see below). This means that if, in those cases, the underestimation of $k_{\text {ic }}$ is due to anharmonic effects, a comparison between $\mathrm{AH}$ and $\mathrm{VH}$ does not seem able to highlight it. Interestingly, broad emission spectra correlate positively with high non-radiative rates.

The choice of the broadening function has only a moderate effect on the radiative rate but, on the contrary, it can have a profound influence on the non-radiative rate. It determines how tightly energy conservation between the initial and final states is enforced. The physical origin of the broadening is twofold: Inhomogeneous broadening (with a Gaussian lineshape) occurs because different molecules in the solution experience slightly different environments leading to small shifts in their energy levels. The energy shifts in an ensemble of molecules follow a Gaussian distribution. These random fluctuations increase with temperature so that the Gaussian broadening can be related to the thermal energy and the solvent reorganization energy. ${ }^{37,38}$ The inhomogeneous broadening can actually be estimated from first principle (using state-specific solvation models ${ }^{38}$ or from molecular dynamics simulations ${ }^{39}$ ) but all these estimates may introduce a computational error. Therefore, due to the scopes of this paper, we prefer to treat is as a phenomenological parameter to better understand its impact on $k_{\mathrm{r}}$ and $k_{\text {ic }}$. In Fig. 4 the vibrationally resolved emission spectra from the $\mathrm{AH}$ model are compared to the experimental spectra. An inhomogeneous broadening of $\mathrm{HWHM}_{\mathrm{G}}=0.125 \mathrm{eV}$ reproduces the spectral width and shape well. However, since the vibrational structure is completely washed out, we use a smaller inhomogeneous broadening of $\mathrm{HWHM}_{\mathrm{G}}=0.02 \mathrm{eV}$ for presenting the emission spectra in Fig. 3. The effect of the inhomogeneous broadening on the radiative and non-radiative rates is only minor.

The homogeneous broadening (Lorentzian lineshape) in turn is due to the finite lifetime $\tau$ of vibrational states, with $\mathrm{HWHM}_{\mathrm{L}}=\gamma=\frac{1}{\tau}$. This type of broadening can be understood as a consequence of the Heisenberg uncertainty principle. Both types of broadening are com- 

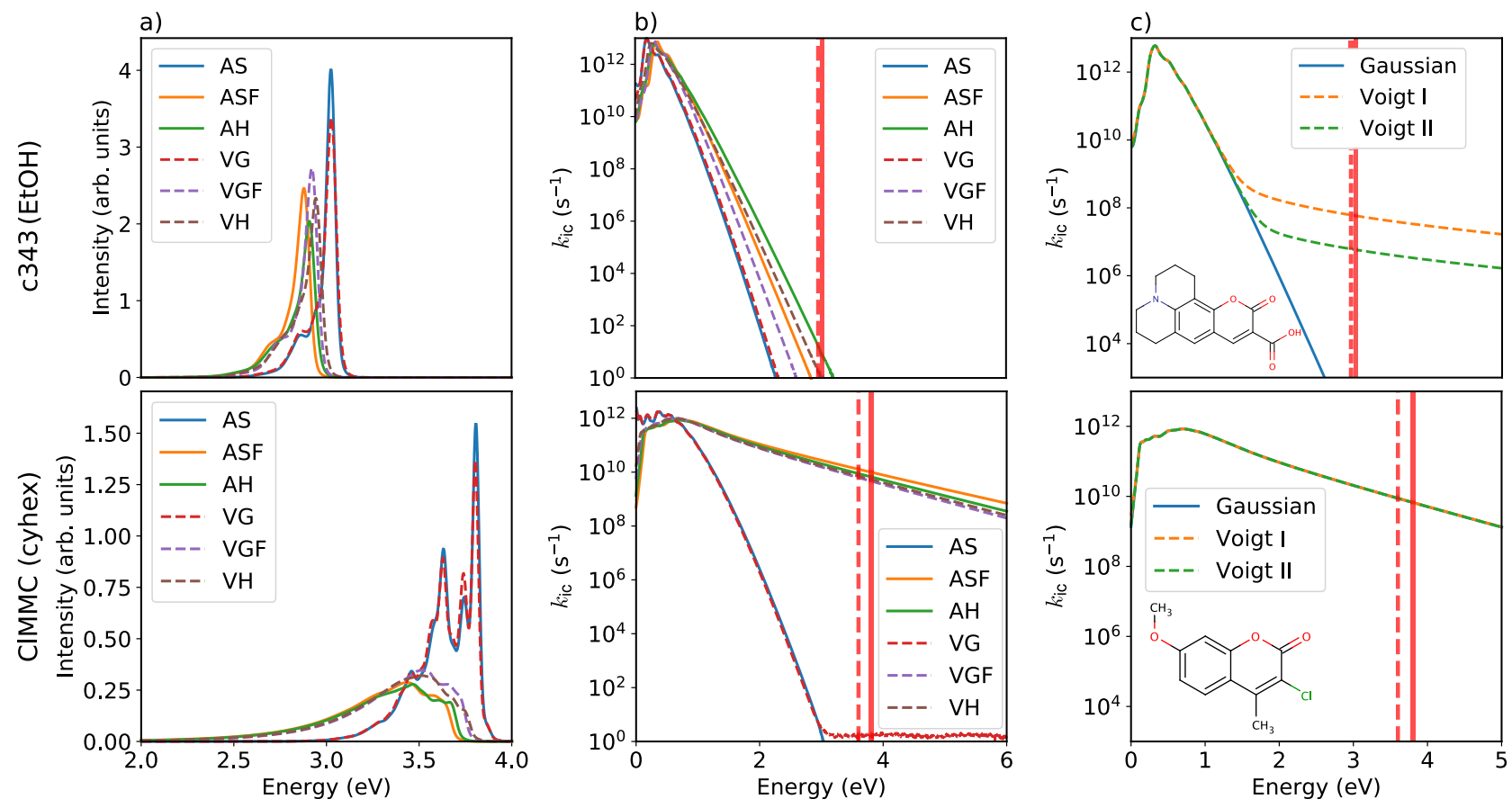

FIG. 3. c343 in EtOH (top) and CIMMC in cyhex (bottom) (at $\mathrm{T}=300 \mathrm{~K}$ ). a) Vibrationally resolved emission spectrum for different harmonic models (with Gaussian broadening of $\mathrm{HWHM}_{\mathrm{G}}=0.02 \mathrm{eV}$. b) IC rate versus emission energy for different models and the same Gaussian broadening. c) IC rate with AH model for different broadening functions: Gaussian $\left(\mathrm{HWHM}_{\mathrm{G}}=\right.$ $0.02 \mathrm{eV})$, Voigt I $\left(\mathrm{HWHM}_{\mathrm{G}}=0.02 \mathrm{eV}, \mathrm{HWHM}_{\mathrm{L}}=0.001 \mathrm{eV}\right)$ and Voigt II $\left(\mathrm{HWHM} \mathrm{G}_{\mathrm{G}}=0.02 \mathrm{eV}, \mathrm{HWHM}_{\mathrm{L}}=0.0001 \mathrm{eV}\right)$. The solid (dashed) red line line marks the adiabatic (vertical) emission energy.

bined in the Voigt profile, which is the convolution of a Gaussian with a Lorentzian function. Data in Table I show that the effect of the adopted Lorentzian broadening on $k_{r}$ is negligible. In contrast, while results for the non-radiative rate are not very sensitive to the Gaussian broadening, they can be dramatically affected by the Lorentzian broadening. A Lorentzian profile has a long tail and thus allows transitions between vibrational states with large energy mismatch, where the Franck-Condon factors are large. Consequently the internal conversion rate increases with $\gamma$ as demonstrated in Fig. 4 of the supplementary material. For large $\gamma$ the rate in fact becomes independent of the vibrational structure and is completely dominated by the broadening function. It is very difficult to estimate $\gamma$ without knowledge of the experimental emission spectrum. If a spectrum is available with some luck a Voigt profile can be fitted to a shoulder of the lowest vibrational peak. However, separating the inhomogeneous from the homogeneous broadening is a tricky task, in particular since both types of broadening affect the spectral width and thus the radiative rate, while it is mostly the homogeneous broadening which affects the internal conversion rate. Also, this approach defeats the purpose of predicting decay rates from first principles. Actually, we compute the contribution to the lifetime due to spontaneous emission as $k_{\mathrm{r}}^{-1}$ (data in Table I). So we can say that $k_{\mathrm{r}}^{-1}$ represents an upper bound for the lifetime. It is usually so long that it does not alter $k_{\mathrm{ic}}$.

The choice of the broadening function affects the nonradiative rates strongly, unless the rate is very large already. The Voigt profile gives a high non-radiative rate at energies where the rate is zero with a Gaussian profile. In this regime the non-radiative rate depends solely on the width of the Lorentzian and not on the vibrational structure. For instance, for coumarin 343 the Gaussian profile predicts $k_{\mathrm{ic}} \approx 0.0 \mathrm{~s}^{-1}$ at an adiabatic emission energy of $3 \mathrm{eV}$, while the Voigt profile predicts $k_{\text {ic }}=10^{7}-10^{9} \mathrm{~s}^{-1}$ depending on the width of the Lorentzian (see top of Fig. $3)$.

In the case of ClMMC (bottom of Fig. 3) accounting for frequency changes (ASF and VGF) increases the rate dramatically by several orders of magnitude relative to the displaced oscillator models. The Duschinsky effect, which in the case of CIMMC is small, increases the rates further. Here adiabatic and vertical models give similar results pointing to the validity of the harmonic approximation. Unlike all other coumarins, the non-radiative rate does not depend on the broadening function, Gaussian and Voigt profiles give the same results over the entire energy range.

We proceed to compare the predicted fluorescence quantum yields, QY $=k_{\mathrm{r}}\left(k_{\mathrm{r}}+k_{\mathrm{ic}}\right)^{-1}$, with experiment in Table I. The large difference between AS/VG and the other models shows that the assumption of independent modes is usually not valid. In Table I we therefore only 
TABLE I. Fluorescence quantum yields QY $=k_{\mathrm{r}}\left(k_{\mathrm{r}}+k_{\mathrm{ic}}\right)^{-1}$ of coumarins predicted by harmonic models. The broadening functions are a Gaussian with $\mathrm{HWHM}_{\mathrm{G}}=0.02 \mathrm{eV}$ and a Voigtian with $\mathrm{HWHM}_{\mathrm{G}}=0.02 \mathrm{eV}$ and $\mathrm{HWHM} \mathrm{L}=0.001 \mathrm{eV}$. $k_{\mathrm{nr}}$ is the experimental non-radiative rate. For caveats about using the Voigt profile see section IV A.

\begin{tabular}{|c|c|c|c|c|c|c|c|c|c|c|c|}
\hline \multirow{2}{*}{$\begin{array}{l}\text { Coumarin } \\
\text { (solvent) }\end{array}$} & \multirow{2}{*}{$\begin{array}{l}\text { Broad. } \\
\text { Funct. }\end{array}$} & \multicolumn{3}{|c|}{$k_{\mathrm{r}} / 10^{8} \mathrm{~s}^{-1}$} & \multicolumn{2}{|c|}{$k_{\mathrm{ic}} / \mathrm{s}^{-1}$} & \multirow{2}{*}{$\frac{k_{\mathrm{nr}} / \mathrm{s}^{-1}}{\text { Exp. }}$} & \multicolumn{3}{|c|}{ QY } & \multirow{2}{*}{ Ref. } \\
\hline & & $\mathrm{AH}$ & $\mathrm{VH}$ & Exp. & $\mathrm{AH}$ & $\mathrm{VH}$ & & $\mathrm{AH}$ & $\mathrm{VH}$ & Exp. & \\
\hline $\begin{array}{l}102 \\
(\mathrm{MeCN})\end{array}$ & $\begin{array}{l}\text { Gauss } \\
\text { Voigt }\end{array}$ & $\begin{array}{l}2.4 \\
2.3\end{array}$ & $\begin{array}{l}2.6 \\
2.6\end{array}$ & 2.8 & $\begin{array}{l}2.1 \cdot 10^{1} \\
4.6 \cdot 10^{7}\end{array}$ & $\begin{array}{l}5.4 \cdot 10^{-1} \\
4.6 \cdot 10^{7}\end{array}$ & $2.7 \cdot 10^{7}$ & $\begin{array}{l}1.00 \\
0.83\end{array}$ & $\begin{array}{l}1.00 \\
0.85\end{array}$ & 0.91 & 29 \\
\hline $\begin{array}{l}102 \\
\text { (cyhex) }\end{array}$ & $\begin{array}{l}\text { Gauss } \\
\text { Voigt }\end{array}$ & $\begin{array}{l}1.9 \\
1.9\end{array}$ & $\begin{array}{l}2.1 \\
2.1\end{array}$ & - & $\begin{array}{l}1.4 \cdot 10^{1} \\
3.2 \cdot 10^{7}\end{array}$ & $\begin{array}{l}2.6 \cdot 10^{-1} \\
3.1 \cdot 10^{7}\end{array}$ & - & $\begin{array}{l}1.00 \\
0.85\end{array}$ & $\begin{array}{l}1.00 \\
0.87\end{array}$ & 1.05 & 29 \\
\hline $\begin{array}{l}102 \\
(\mathrm{EtOH})\end{array}$ & $\begin{array}{l}\text { Gauss } \\
\text { Voigt }\end{array}$ & $\begin{array}{l}2.3 \\
2.3\end{array}$ & $\begin{array}{l}2.6 \\
2.6\end{array}$ & 1.3 & $\begin{array}{l}2.0 \cdot 10^{1} \\
4.6 \cdot 10^{7}\end{array}$ & $\begin{array}{l}5.3 \cdot 10^{-1} \\
4.6 \cdot 10^{7}\end{array}$ & $8.6 \cdot 10^{7}$ & $\begin{array}{l}1.00 \\
0.84\end{array}$ & $\begin{array}{l}1.00 \\
0.85\end{array}$ & $0.60,0.77$ & 30,31 \\
\hline $\begin{array}{l}\mathbf{3 4 3} \\
(\mathrm{EtOH})\end{array}$ & $\begin{array}{l}\text { Gauss } \\
\text { Voigt }\end{array}$ & $\begin{array}{l}3.0 \\
2.9\end{array}$ & $\begin{array}{l}3.4 \\
3.4\end{array}$ & - & $\begin{array}{l}7.9 \cdot 10^{0} \\
5.8 \cdot 10^{7}\end{array}$ & $\begin{array}{l}9.6 \cdot 10^{-1} \\
6.1 \cdot 10^{7}\end{array}$ & - & $\begin{array}{l}1.00 \\
0.83\end{array}$ & $\begin{array}{l}1.00 \\
0.85\end{array}$ & $0.81,0.63$ & 32,34 \\
\hline $\begin{array}{l}\mathbf{5 2 2} \\
(\mathrm{MeCN})\end{array}$ & $\begin{array}{l}\text { Gauss } \\
\text { Voigt }\end{array}$ & $\begin{array}{l}1.7 \\
1.7\end{array}$ & $\begin{array}{l}2.0 \\
1.9\end{array}$ & 1.5 & $\begin{array}{l}4.3 \cdot 10^{3} \\
6.4 \cdot 10^{7}\end{array}$ & $\begin{array}{l}6.9 \cdot 10^{2} \\
6.3 \cdot 10^{7}\end{array}$ & $2.3 \cdot 10^{7}$ & $\begin{array}{l}1.00 \\
0.73\end{array}$ & $\begin{array}{l}1.00 \\
0.75\end{array}$ & 0.87 & 29 \\
\hline $\begin{array}{l}\mathbf{5 2 2} \\
\text { (cyhex) }\end{array}$ & $\begin{array}{l}\text { Gauss } \\
\text { Voigt }\end{array}$ & $\begin{array}{l}1.4 \\
1.4\end{array}$ & $\begin{array}{l}1.6 \\
1.6\end{array}$ & 2.3 & $\begin{array}{l}4.0 \cdot 10^{4} \\
4.3 \cdot 10^{7}\end{array}$ & $\begin{array}{l}1.3 \cdot 10^{4} \\
4.3 \cdot 10^{7}\end{array}$ & $5.0 \cdot 10^{6}$ & $\begin{array}{l}1.00 \\
0.76\end{array}$ & $\begin{array}{l}1.00 \\
0.79\end{array}$ & 0.98 & 29 \\
\hline $\begin{array}{l}\text { ClMMC } \\
\text { (cyhex) }\end{array}$ & $\begin{array}{l}\text { Gauss } \\
\text { Voigt }\end{array}$ & $\begin{array}{l}0.7 \\
0.7\end{array}$ & $\begin{array}{l}1.2 \\
1.2\end{array}$ & 1.9 & $\begin{array}{l}6.6 \cdot 10^{9} \\
6.7 \cdot 10^{9}\end{array}$ & $\begin{array}{l}5.1 \cdot 10^{9} \\
5.2 \cdot 10^{9}\end{array}$ & $1.4 \cdot 10^{9}$ & $\begin{array}{l}0.01 \\
0.01\end{array}$ & $\begin{array}{l}0.02 \\
0.02\end{array}$ & 0.12 & 33 \\
\hline $\begin{array}{l}\text { ClMMC } \\
\text { (water) }\end{array}$ & $\begin{array}{l}\text { Gauss } \\
\text { Voigt }\end{array}$ & $\begin{array}{l}2.6 \\
2.6\end{array}$ & $\begin{array}{l}2.9 \\
2.9\end{array}$ & 2.1 & $\begin{array}{l}4.0 \cdot 10^{7} \\
7.9 \cdot 10^{7}\end{array}$ & $\begin{array}{l}1.4 \cdot 10^{7} \\
5.2 \cdot 10^{7}\end{array}$ & $6.0 \cdot 10^{7}$ & $\begin{array}{l}0.87 \\
0.76\end{array}$ & $\begin{array}{l}0.95 \\
0.85\end{array}$ & 0.83 & 33 \\
\hline
\end{tabular}

list rates and quantum yields for the $\mathrm{AH}$ and $\mathrm{VH}$ models. With the exception of CIMMC, the Gaussian profile yields non-radiative rates which are orders of magnitude too low, so that the fluorescence quantum yield is always 1. With a Voigt profile, the non-radiative rates increase to a reasonable level, however, they depend on the arbitrary choice of the width of the Lorentzian. The apparent agreement between the experimental and theoretical rates when using the Voigt profile with $\gamma=0.001 \mathrm{eV}$ can be misleading. If we interpret the homogeneous broadening as the result of a finite lifetime of the excited state, this $\gamma$ corresponds to a lifetime of 658 fs, i.e. to the existence of another non-radiative decay processes (e.g. ISC, $\mathrm{CI})$ that has $k_{\mathrm{nr}}^{\prime}=1.5 \times 10^{12} \mathrm{~s}^{-1}$. If this process exists, it completely dominates the other ones and brings $\mathrm{QY}$ to 0 , since QY $=k_{\mathrm{r}}\left(k_{\mathrm{r}}+k_{\text {ic }}+k_{\mathrm{nr}}^{\prime}\right)^{-1}$. In other terms, the fact that the internal conversion rate increases from $10^{1}$ to $10^{7} \mathrm{~s}^{-1}$ is of little interest. No excited state population decays via internal conversion, since the other process is much faster. Before internal conversion can set in, all population has already decayed via the additional channel. Thus, if a large $\gamma$ is needed to reproduce experimental rates, this means that either the most important decay channel is missing in the model or that anharmonicities are not negligible. Therefore fitting $\gamma$ to reproduce experimental non-radiative $\operatorname{rates}^{40}$ is not advisable.

A possible way to determine a unique $\gamma$ is to use a selfconsistent procedure: We start with an arbitrary $\gamma$ and compute the radiative and IC rates. In the following iterations, we set $\gamma=k_{\mathrm{r}}+k_{\mathrm{ic}}$ and repeat the calculation of the rates with the new Lorentzian broadening. $\gamma$ converges typically after a few iterations to a unique value that does not depend on the initial starting value. The self-consistent broadenings are close to 0: $\quad \gamma_{\mathrm{SC}}=4.4 \times 10^{-6} \mathrm{eV}$ for ClMMC in cyhex and $\gamma_{\mathrm{SC}}=1.0 \times 10^{-7} \mathrm{eV}$ for $\mathbf{3 4 3}$ in ethanol. Although substituting the results of first-order perturbation theory back into a first-order theory is somewhat questionable, this procedure shows that, if we require $\gamma$ to be uniquely defined, its value is much smaller than what would be necessary to reproduce experimental non-radiative rates.

Only for ClMMC in water and cyclohexane (cyhex) the quantum yield is predicted reliably.

We can identify two conditions that should be met if the results are to be trusted: (1) $\mathrm{AH}$ and $\mathrm{VH}$ models should agree and (2) Gaussian and Voigt profiles should not differ greatly at the adiabatic emission energy. There is also an apparent connection between the width of the emission spectrum and the robustness of the predicted rates to the choice of the broadening function.

For systems with short vibronic progressions (narrow spectra), such as coumarins 102, 343 and 522, the rate is small and very sensitive to the broadening, while for those with long vibronic progressions (broad spectra), such as CIMMC in cyhex and to a lesser degree in water, all broadening functions give the same result.

One of the decay processes we have neglected so far is intersystem crossing from $\mathrm{S}_{1}$ to the energetically close $\mathrm{T}_{2}$ state (see Table 1 in the $\mathrm{SI}$ ). Although the spin-orbit couplings are very small, we cannot exclude that this decay channel contributes significantly to the non-radiative rate. We leave this question to a future study. In a relativistic treatment, where spin is not a good quantum number anymore, singlet and triplet states would be mixed, so that the distinction between IC and ISC 
becomes blurred. Therefore all of the above considerations would also apply to ISC. However, we expect the harmonic approximation to be better justified in the case of ISC, since the energy gap is usually smaller.

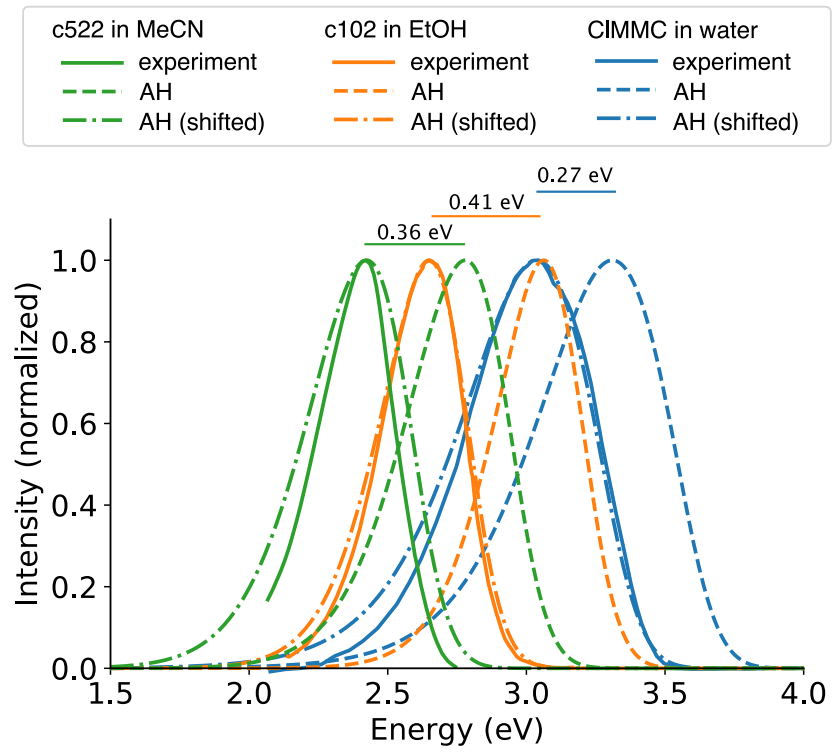

FIG. 4. Comparison between experimental and theoretical emission spectra. The theoretical spectra were obtained with the AH model $\left(\mathrm{HWHM}_{\mathrm{G}}=0.125 \mathrm{eV}\right.$ and $\left.\mathrm{HWHM}_{\mathrm{L}}=0 \mathrm{eV}\right)$ at $\mathrm{T}=300 \mathrm{~K}$. To make comparison of the spectral width and shape easier, the theoretical spectra were shifted so that the maxima of the experimental and theoretical curves coincide. Experimental spectra were digitized from the following sources: c522 in $\mathrm{MeCN}^{41}, \mathbf{c 1 0 2}$ in $\mathrm{EtOH}^{30}, \mathbf{C l M M C}$ in water $^{33}$.

\section{B. Anharmonic AS model - Anharmonicity and Broadening function}

Finally we consider the effects of anharmonicity and the choice of the broadening function on the internal conversion rate in the adiabatic shift model. We use the coumarin CIMMC in cyhex as an example.

Figure 5 depicts $k_{\text {ic }}(\Delta E)$ for increasing anharmonicity. At intermediate energies $(0.5<\Delta E<3.0 \mathrm{eV})$ the anharmonic rates are higher than the harmonic one, but $\log \left(k_{\text {ic }}\right)$ still decreases linearly with $\Delta E$, while at higher energies $(3.0<\Delta E)$ the rates start to deviate from the exponential energy gap law ${ }^{42}$. High internal conversion rates require energy conservation and large Franck-Condon factors, but these two conditions cannot be fulfilled at the same time: If the final states are low-lying vibrational states, the Franck-Condon factors are high but energy conservation is poorly fulfilled. On the other hand, if the final states are highly excited vibrational states, such that the sum of the vibrational energies equals the optical gap $\Delta E$, the Franck-Condon factors are very low, due to the oscillatory nature of high- lying vibrational states. In the harmonic approximation, the Franck-Condon factors $\left|\left\langle n^{\prime} \mid m\right\rangle\right|^{2}$ vanish quickly as $m \rightarrow \infty$. The effect of including anharmonicity is that the Franck-Condon factors decrease slower with increasing $m$ (see Fig. 2 in the supplementary material). Therefore more final states are available which have relatively high overlaps with the initial states in the anharmonic case than in the harmonic case.

What value should one take for the anharmonicity $\chi$ ? A possible qualitative argument for selecting a plausible value for $\chi$ is the following: In infrared spectroscopy it is rather customary to multiply the computed harmonic frequencies by a factor, usually 0.96 for B3LYP $/ 6-31 G(d)^{43}$, since this improves the comparison of the harmonic vibrational frequencies with the experimental fundamentals. Of course this is just a phenomenological parameter and it corrects also for other errors but it is considered that one of the most important effects is anharmonicity. ${ }^{43}$ According to eqn. 13 the ratio of the first transition energy and the harmonic frequency is

$$
\frac{\epsilon_{1}-\epsilon_{0}}{\hbar \omega}=1-2 \chi
$$

Therefore if we impose that this fraction is 0.96 we get $\chi=0.02$. This suggests that the values $\chi=$ $0.03, \ldots, 0.07$, where the deviations of $k_{\text {ic }}$ from the harmonic approximation are sizeable, might be too large. On the other hand, the anharmonic AS model just includes diagonal anharmonicities, while for the harmonic models we have seen that quadratic couplings (frequency changes and Duschinsky effect) play an important role. Therefore it is conceivable that the off-diagonal anharmonicities would be even more important than the diagonal ones.

These simple calculations with the AS model show that deviations from the energy gap law are attributable to anharmonicities. Choosing a large Lorentzian broadening $\gamma$ has a similar effect (cf. Fig. 4 in the SI). However, a large $\gamma$ actually implies the existence of a non-radiative process we have not considered which reduces the lifetime of the excited states. In both cases (large $\chi$ or large $\gamma$ ) the rates are higher and the function $\log \left(k_{\text {ic }}(\Delta E)\right)$ exhibits a kink where the slope of the curve suddenly becomes less steep.

Furthermore, it should be noted that the choice of the method to describe the electronic structure adds another uncertainty to the computed rates. Even within density functional theory, the choice of the functional type (GGA, hybrid, range-corrected hybrid) and the solvation model can lead to differences in the vibrational structure and, above all, to deviations in the vertical/adiabatic energies in the order of $0.5 \mathrm{eV}$. The differences in the resulting emission energies and IC rates between the PBE0 and the B3LYP functional are shown in Table II. Since the slope of the curve $k_{\text {ic }}$ vs. $\Delta E$ is very steep, the choice of the functional can change the internal conversion rates by several orders of magnitude. 


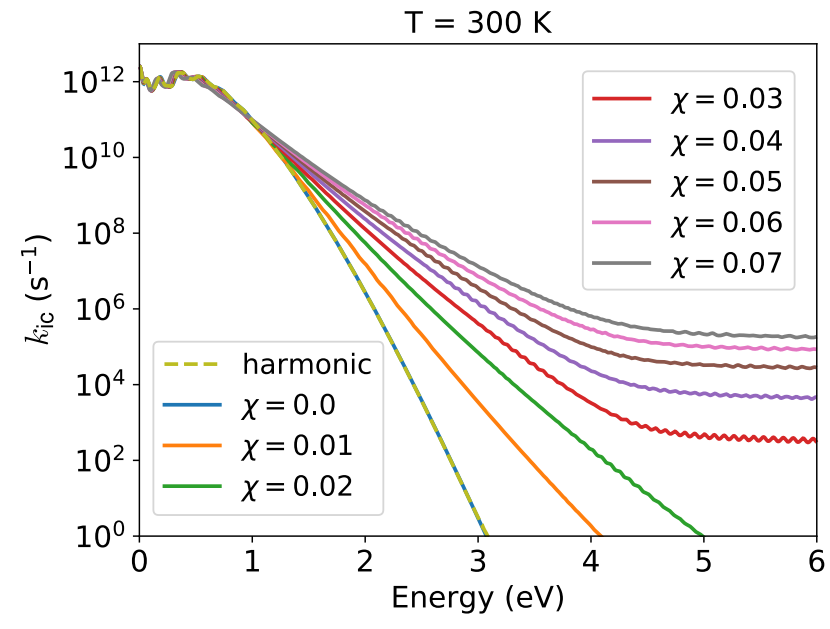

FIG. 5. CIMMC in cyhex. Internal conversion rates from anharmonic AS model for different degrees of anharmonicity $\chi$. The dashed line shows the rate obtained with FCclasses 3 for the AS model, which should be identical to the $\chi=0.0$ case.

TABLE II. Emission energies and internal conversion rates for the functionals PBE0 and B3LYP. $E_{\text {em }}$ is the energy at the maximum of the emission spectrum. The theoretical spectra were obtained with the $\mathrm{AH}$ model $\left(\mathrm{HWHM}_{\mathrm{G}}=0.125 \mathrm{eV}\right.$ and $\mathrm{HWHM}_{\mathrm{L}}=0 \mathrm{eV}$ ) at $\mathrm{T}=300 \mathrm{~K}$. Sources for experimental spectra are the same as in Fig. 4, except for coumarin $\mathbf{3 4 3}^{44}$ (PhotochemCAD online database).

\begin{tabular}{|c|c|c|c|c|c|}
\hline \multirow{2}{*}{$\begin{array}{l}\text { Coumarin } \\
\text { (solvent) }\end{array}$} & \multicolumn{3}{|c|}{$E_{\mathrm{em}} / \mathrm{eV}$} & \multicolumn{2}{|c|}{$k_{\mathrm{ic}}\left(\mathrm{s}^{-1}\right)$} \\
\hline & PBE0 & B3LYP & exp. & PBE0 & B3LYP \\
\hline $102(\mathrm{MeCN})$ & 3.06 & 2.63 & - & & \\
\hline 102 (cyhex) & 26 & 2.99 & - & $1.4 \cdot 10^{1}$ & $9.0 \cdot 10^{2}$ \\
\hline $102(\mathrm{EtOH})$ & .06 & 2.64 & 2.65 & $2.0 \cdot 10^{1}$ & $3.9 \cdot 10^{3}$ \\
\hline $343(\mathrm{EtOH})$ & 2.88 & 2.77 & 2.65 & $7.9 \cdot 10^{0}$ & $1.5 \cdot 10^{2}$ \\
\hline $522(\mathrm{MeCN})$ & 2.78 & 2.39 & 2.42 & $4.3 \cdot 10^{3}$ & $4.6 \cdot 10^{5}$ \\
\hline 522 (cyhex) & .99 & 2.70 & - & $4.0 \cdot 10^{4}$ & $3.8 \cdot 10^{5}$ \\
\hline CIMMC (cyhex & 3.43 & 3.40 & - & $6.6 \cdot 10^{9}$ & $1.2 \cdot 10^{10}$ \\
\hline ClMMC (water) & 3.31 & 3.32 & 3.04 & $4.0 \cdot 10^{7}$ & $3.0 \cdot 10^{7}$ \\
\hline
\end{tabular}

\section{v. CONCLUSION}

In this work we investigated the reliability of the harmonic approximation for the prediction of the radiative and non-radiative decay rates and therefore of the fluorescence quantum yield of a number of coumarin dyes. We first examined the performance of a hierarchy of vertical and adiabatic harmonic models and, afterward we developed and applied a simple AS anharmonic model in order to qualitatively analyze the possible effect of anharmonicity. Therefore we can try to enunciate a number of "rules of thumb" that can help judging whether the results predicted from harmonic models are reliable:

(Rule I) Harmonic predictions for $k_{r}$ are in good agreement with experiment for the few cases where experimen- tal data is available. Frequency changes and Duschinsky mixings have only a moderate effect and tend to slightly decrease $\mathrm{k}_{\mathrm{r}}$. (Rule II) As far as the prediction of $k_{i c}$ is concerned, it is fundamental to account for frequency changes and Duschinsky rotation, since they increase $k_{i c}$ strongly. In the examples considered the Duschinsky effect is more moderate, but this is a peculiarity of coumarins, and there are many other systems where mode mixing can be significant. Therefore we advise against using the AS and VG models, as they neglect the effects that contribute most to the non-radiative rate. $k_{\text {ic }}$ shows a much larger dependence on the specificity of the molecule and the adopted methodology than $k_{\mathrm{r}}$. Therefore, it is much more difficult to compute. This is an expected result since $k_{\text {ic }}$ mainly depends on a reliable description of strongly excited vibrational states, i.e. the same states that are responsible for the very far tail of the emission spectrum. (Rule III) As a matter of fact a broad emission spectrum correlates with large $k_{\mathrm{ic}}$. These observations suggest that a further check of the reliability of computational estimates might be: Do the calculations allow for a realistic reproduction of the low-energy tail of the emission spectrum?

Broadening functions insert phenomenologically those effects that attenuate the energy-conservation rule. They account for important facts like the effect of energy fluctuations, lifetimes and other effects that matter. (Rule IV) The inhomogeneous broadening has a weak impact on $k_{\mathrm{r}}$ and practically no impact on $k_{\mathrm{ic}}$. The homogeneous broadening is connected with the lifetime of the excited state, which is equal or shorter than the fluorescence lifetime $k_{\mathrm{r}}^{-1}$. Of course, other processes can decrease the lifetime so that we also treated $\gamma$ as a parameter. However, since in general we are not able to compute a realistic number for the lifetime, we use this analysis only to set a limit on the reliability of our results: (Rule V) If our results strongly depend on $\gamma$ they are not reliable. In fact in those cases, $k_{\text {ic }}$ is not dominated by the vibronic profile (which carries information on the target molecule) but by the phenomenological width of the Lorentzian $\gamma$. This happens when, with our level of theory, we predict a very small $k_{\text {ic }}$. It may be that in reality the non-radiative decay is much faster than what we predict but this depends on other processes - conical intersections, intersystem crossing, internal conversions due to interactions with the environment (a kind of pressure broadening) that have not been considered in our calculations. They would strongly modify the prediction of the fluorescence quantum yield and, clearly, their effect cannot be reproduced by a phenomenological increase of $\gamma$ in the computation of the non-radiative process we have considered. In summary, when they are important they should be identified and properly taken into account. This clearly makes a reliable computational prediction of QY for these systems much more complicated.

The effect of anharmonicity (beyond the AS model) is the most delicate and elusive point, since anharmonicities can be many. In principle, conical intersections and 
multiple conformations, all fall into the category of extreme anharmonicities. Assuming Morse potentials for all modes, we find that for a typical value of $\chi=0.02$ anharmonic effects are not drastic. One should bear in mind, however, that a Morse potential does not describe low energy modes such as rotations around single bonds correctly, and that the coupling between different modes is also neglected in the AS model. Including anharmonic corrections for a realistic model of coupled modes is not feasible so far.

(Rule VI) An obvious condition is that results from $A H$ and VH models must be similar, as this is necessary for the validity of the harmonic approximation. Since we should expect that the error is at least equal to the difference of $\mathrm{AH}$ and $\mathrm{VH}$ predictions, this sets a lower bound for the accuracy. Our results however clarify that is only a necessary and not a sufficient conditions. A second condition is that small values of the Lorentzian broadening should not strongly modify our estimate of the QY.

(Rule VII) Interestingly, our results suggest that the width of the Lorentzian function and anharmonic effects start ruling $k_{i c}$ for values of $\Delta E$ for which $k_{\text {ic }}(\Delta E)<10^{-5} k_{\text {ic }}^{\max }$. In summary we can conclude that our methodology can be considered reliable only for molecules with adiabatic excitation energies such that $k_{\text {ic }}(\Delta E)>10^{-5} k_{\text {ic }}^{\max }$. Since $k_{\text {ic }}^{\max }$ is typically $10^{12}-$ $10^{13} \mathrm{~s}^{-1}$ the critical values for which our computed nonradiative rates start not being robust anymore are $10^{7}-$ $10^{8} \mathrm{~s}^{-1}$, i.e. the typical values for $k_{\mathrm{r}}$. This limits our predictability to the extreme case $\mathrm{QY}=0$ : If $k_{\text {ic }} \gg k_{\mathrm{r}}$ already in the harmonic approximation, the prediction $\mathrm{QY}=0$ is very robust, since anharmonicity and homogeneous broadening only increase the internal conversion rate. However, values of, QY $>0$, are likely those more challenging for our theory and in those cases our prediction will always be a little bit questionable.

The fulfillment of the condition $k_{\mathrm{ic}}(\Delta E)>10^{-5} k_{\mathrm{ic}}^{\max }$ depends on two factors working in different directions: the value of $\Delta E$, and the extension of the vibronic progressions. The smaller $\Delta E$ and the longer the vibronic progressions, the easier it is that the condition is true. Small $\Delta E$ means emission in the red region of visible spectrum or in the near infrared. Long vibronic progressions means molecules with broad emission spectra and therefore characterized by significant displacements of the excited- and ground-state equilibrium geometries and, according to our analysis, also significant changes in the normal mode frequencies. It is worthwhile to reiterate the hypotheses underlying these considerations: We assume that direct internal conversion from a thermalized bright state to the ground state, triggered by nonadiabatic couplings, is the only relevant non-radiative process. In other words, sequential decay, possible ISC, photoisomerizations and decay at conical intersections are neglected.

The chosen examples illustrate that in order to make theoretical predictions for fluorescence quantum yields, it is not sufficient to compare only simulated quantum yields with the experimental ones, but it is recommendable to compare and analyze the radiative and non-radiative rates and the influence of varying the harmonic model and broadening parametrization. If there is a drastic difference in the obtained values for $k_{\text {ic }}$ one should not consider these results accurate enough for the description of fluorescence quantum yields. In conclusion, a "blackbox" method for predicting fluorescence quantum yields from first principles does not seem within reach, yet.

\section{SUPPLEMENTARY MATERIAL}

See supplementary material for additional figures mentioned in the text.

\section{ACKNOWLEDGMENTS}

$\mathrm{RM}$ and $\mathrm{AH}$ acknowledge the financial support within the ERC Consolidator Grant "DYNAMO" (Grant No. 646737). RM and JH thank the Deutsche Forschungsgemeinschaft for support of this work within the Research Group FOR 1809. This research was partially supported by the project STIM - REI, Contract Number: KK.01.1.1.01.0003, funded by the European Union through the European Regional Development Fund - the Operational Programme Competitiveness and Cohesion 2014-2020 (KK.01.1.1.01). VBK and MB acknowledge computational facilities of the supercomputer SRCE at University of Zagreb as well as Doctoral study of Biophysics at the University of Split.

${ }^{1}$ J. Batchelder, A. Zewai, and T. Cole, Appl. Optics 18, 3090 (1979).

${ }^{2}$ J. V. Frangioni, Curr. Opin. Chem. Biol. 7, 626 (2003).

${ }^{3}$ Y. Niu, W. Li, Q. Peng, H. Geng, Y. Yi, L. Wang, G. Nan, D. Wang, and Z. Shuai, Mol. Phys. 116, 1078 (2018).

${ }^{4}$ F. Santoro and J. Cerezo, FCclasses3, a code to simulate electronic spectra. Version FCclasses3-0.1, 2019. Visit http://www.pi.iccom.cnr.it/fcclasses.

${ }^{5} \mathrm{~S}$. Banerjee, A. Baiardi, J. Bloino, and V. Barone, J. Chem. Theor. Comput. 12, 774 (2016).

${ }^{6}$ R. R. Valiev, V. N. Cherepanov, R. T. Nasibullin, D. Sundholm, and T. Kurten, Phys. Chem. Chem. Phys. 21, 18495 (2019).

${ }^{7}$ B. de Souza, G. Farias, F. Neese, and R. Izsák, J. Chem. Theor. Comput. 15, 1896 (2019).

${ }^{8}$ R. R. Valiev, V. N. Cherepanov, G. V. Baryshnikov, and D. Sundholm, Phys. Chem. Chem. Phys. 20, 6121 (2018).

${ }^{9}$ Q. Peng, Y. Yi, Z. Shuai, and J. Shao, J. Am. Chem. Soc. 129, 9333 (2007).

${ }^{10}$ A. W. Kohn, Z. Lin, and T. Van Voorhis, J. Phys. Chem. C (2019).

${ }^{11}$ J. Hoche, A. Schulz, L. M. Dietrich, A. Humeniuk, M. Stolte, D. Schmidt, T. Brixner, F. Würthner, and R. Mitrić, Chem. Sci. 10, 11013 (2019).

${ }^{12}$ S. Lin, C. Chang, K. Liang, R. Chang, J. Zhang, T. Yang, M. Hayashi, Y. Shiu, and F. Hsu, Adv. Chem. Phys. 121, 1 (2002).

${ }^{13}$ Q. Peng, Y. Niu, C. Deng, and Z. Shuai, Chem. Phys. 370, 215 (2010).

${ }^{14}$ F. J. Avila Ferrer and F. Santoro, Phys. Chem. Chem. Phys. 14, 13549 (2012). 
${ }^{15}$ The effect of anharmonicity on the absorption and fluorescence spectra has been studied in Ref. ${ }^{45}$ also employing the displaced oscillator model (AS) and the Morse potential.

${ }^{16}$ Y. Niu, Q. Peng, and Z. Shuai, Sci. China Ser. B 51, 1153 (2008).

${ }^{17}$ Q. Peng, Y. Niu, Q. Shi, X. Gao, and Z. Shuai, J. Chem. Theor. Comput. 9, 1132 (2013).

${ }^{18}$ P. M. Morse, Phys. Rev. 34, 57 (1929).

${ }^{19}$ J. P. Dahl and M. Springborg, J. Chem. Phys. 88, 4535 (1988).

${ }^{20} \mathrm{C}$. Adamo and V. Barone, J. Chem. Phys. 110, 6158 (1999).

${ }^{21}$ M. Ernzerhof and G. E. Scuseria, J. Chem. Phys. 110, 5029 (1999).

22 A. D. Becke, J. Chem. Phys. 98 (1993).

${ }^{23}$ F. Weigend, Phys. Chem. Chem. Phys. 8, 1057 (2006).

${ }^{24}$ M. J. Frisch, G. W. Trucks, H. B. Schlegel, G. E. Scuseria, M. A. Robb, J. R. Cheeseman, G. Scalmani, V. Barone, G. A. Petersson, H. Nakatsuji, X. Li, M. Caricato, A. V. Marenich, J. Bloino, B. G. Janesko, R. Gomperts, B. Mennucci, H. P. Hratchian, J. V. Ortiz, A. F. Izmaylov, J. L. Sonnenberg, D. WilliamsYoung, F. Ding, F. Lipparini, F. Egidi, J. Goings, B. Peng, A. Petrone, T. Henderson, D. Ranasinghe, V. G. Zakrzewski, J. Gao, N. Rega, G. Zheng, W. Liang, M. Hada, M. Ehara, K. Toyota, R. Fukuda, J. Hasegawa, M. Ishida, T. Nakajima, Y. Honda, O. Kitao, H. Nakai, T. Vreven, K. Throssell, J. A. Montgomery, Jr., J. E. Peralta, F. Ogliaro, M. J. Bearpark, J. J. Heyd, E. N. Brothers, K. N. Kudin, V. N. Staroverov, T. A. Keith, R. Kobayashi, J. Normand, K. Raghavachari, A. P. Rendell, J. C. Burant, S. S. Iyengar, J. Tomasi, M. Cossi, J. M. Millam, M. Klene, C. Adamo, R. Cammi, J. W. Ochterski, R. L. Martin, K. Morokuma, O. Farkas, J. B. Foresman, and D. J. Fox, "Gaussian 16 Revision A.03," (2016), gaussian Inc. Wallingford CT.

${ }^{25}$ F. J. A. Ferrer, J. Cerezo, J. Soto, R. Improta, and F. Santoro, Comput. Theor. Chem. 1040-1041, 328 (2014).

${ }^{26}$ V. Barone, M. Biczysko, J. Bloino, L. Carta, and A. Pedone, Comput. Theor. Chem. 1040-1041, 144 (2014).
${ }^{27}$ J. Tomasi, B. Mennucci, and R. Cammi, Chem. Rev. 105, 2999 (2005).

${ }^{28} \mathrm{M}$. Taniguchi and J. S. Lindsey, Photochem. and Photobiol. 94, 290 (2018).

${ }^{29}$ G. Jones II, W. R. Jackson, C. Y. Choi, and W. R. Bergmark, J. Phys. Chem. 89, 294 (1985).

${ }^{30}$ S. Mondal, R. Halder, B. Biswas, B. Jana, and P. C. Singh, J. Chem. Phys. 144, 184504 (2016).

${ }^{31}$ K. Rurack and M. Spieles, Anal. Chem. 83, 1232 (2011).

${ }^{32}$ V. A. Lapina, T. A. Pavich, P. P. Pershukevich, A. V. Trofimov, N. N. Trofimova, Y. B. Tsaplev, and P. P. Zak, J. Phys. Org. Chem. 30, e3731 (2017).

${ }^{33}$ J. S. Seixas de Melo, R. S. Becker, and A. L. Maqanita, J. Phys. Chem. 98, 6054 (1994).

${ }^{34}$ G. Reynolds and K. H. Drexhage, Opt. Comm. 13, 222 (1975).

${ }^{35}$ J. Cerezo, F. J. A. Ferrer, and F. Santoro, Phys. Chem. Chem. Phys. 17, 11401 (2015).

${ }^{36}$ C. M. Krauter, J. Möhring, T. Buckup, M. Pernpointner, and M. Motzkus, Phys. Chem. Chem. Phys. 15, 17846 (2013).

${ }^{37}$ R. Marcus, J. Chem. Phys. 43, 1261 (1965).

${ }^{38}$ F. J. A. Ferrer, R. Improta, F. Santoro, and V. Barone, Phys. Chem. Chem. Phys. 13, 17007 (2011).

${ }^{39}$ J. Cerezo, F. J. A. Ferrer, G. Prampolini, and F. Santoro, J. Chem. Theory Comput. 11, 5810 (2015).

${ }^{40}$ I. Kim, W.-J. Son, Y.-S. Choi, A. Osipov, D. Lee, H. Lee, Y. Jung, J. Son, H. Choi, W. Sotoyama, et al., J. Chem. Phys. C 123, 11140 (2019).

${ }^{41}$ S. Nad and H. Pal, J. Photochem. Photobiol. A 134, 9 (2000).

${ }^{42}$ R. Englman and J. Jortner, Mol. Phys. 18, 145 (1970).

${ }^{43}$ A. P. Scott and L. Radom, J. Phys. Chem. 100, 16502 (1996).

${ }^{44}$ H. Du, R.-C. A. Fuh, J. Li, L. A. Corkan, and J. S. Lindsey, Photochemistry and photobiology 68, 141 (1998).

${ }^{45}$ C. Zhu, K. K. Liang, M. Hayashi, and S. H. Lin, Chem. Phys. 358, 137 (2009). 